\title{
The dynamics of methicillin-resistant Staphylococcus aureus exposure in a hospital model and the potential for environmental intervention
}

\author{
Nottasorn Plipat, lan H Spicknall, James S Koopman and Joseph NS Eisenberg
}

\begin{abstract}
Background: Methicillin-resistant Staphylococcus aureus (MRSA) is a major cause of healthcare-associated infections. An important control strategy is hand hygiene; however, non-compliance has been a major problem in healthcare settings. Furthermore, modeling studies have suggested that the law of diminishing return applies to hand hygiene. Other additional control strategies such as environmental cleaning may be warranted, given that MRSA-positive individuals constantly shed contaminated desquamated skin particles to the environment.

Methods: We constructed and analyzed a deterministic environmental compartmental model of MRSA fate, transport, and exposure between two hypothetical hospital rooms: one with a colonized patient, shedding MRSA; another with an uncolonized patient, susceptible to exposure. Healthcare workers (HCWs), acting solely as vectors, spread MRSA from one patient room to the other.

Results: Although porous surfaces became highly contaminated, their low transfer efficiency limited the exposure dose to HCWs and the uncolonized patient. Conversely, the high transfer efficiency of nonporous surfaces allows greater MRSA transfer when touched. In the colonized patient's room, HCW exposure occurred more predominantly through the indirect (patient to surfaces to HCW) mode compared to the direct (patient to HCW) mode. In contrast, in the uncolonized patient's room, patient exposure was more predominant in the direct (HCW to patient) mode compared to the indirect (HCW to surfaces to patient) mode. Surface wiping decreased MRSA exposure to the uncolonized patient more than daily surface decontamination. This was because wiping allowed higher cleaning frequency and cleaned more total surface area per day.

Conclusions: Environmental cleaning should be considered as an integral component of MRSA infection control in hospitals. Given the previously under-appreciated role of surface contamination in MRSA transmission, this intervention mode can contribute to an effective multiple barrier approach in concert with hand hygiene.
\end{abstract}

\section{Background}

Methicillin-resistant Staphylococcus aureus (MRSA) has become an important cause of healthcare-associated infections in the U.S. and worldwide [1-3]. An important strategy for preventing healthcare-associated infection (HAI), including MRSA infections, has been hand hygiene [4-8]. The role of hand hygiene in decreasing HAIs was established in the $1840 \mathrm{~s}$, when Semmelweis demonstrated that hand scrubbing with chlorinated lime solution resulted in a drastic decrease in maternal mortality due to puerperal sepsis [9]. A study in 1962, also showed that

* Correspondence: nplipat@umich.edu

Department of Epidemiology, School of Public Health, University of Michigan, Ann Arbor, Michigan, USA

\section{Ciomed Central}

newborns, cared for by nurses who did not perform hand hygiene, were more likely to acquire $S$. aureus infection than those cared for by nurses who performed hand hygiene [10]. Although these historical studies as well as many intervention studies have demonstrated the efficacy of hand hygiene, the evidence of benefit has not always been translated into routine practice [11,12]. Hand hygiene noncompliance remains a major problem in healthcare settings [11]. Furthermore, studies have suggested that the law of diminishing return applies to hand hygiene, with the greatest benefits occurring in the first $20 \%$ of compliance. The additional benefits of hand hygiene decrease as the baseline compliance levels increase $[13,14]$. 
Prevention of MRSA infections will likely need more than hand hygiene intervention; this is partly because of poor compliance, and also because of possibility of recontamination of hands from surface as well as crosscontamination from the skin [15-19]. Due to these limitations, interventions performed in concert with hand hygiene may be important to further decrease MRSA HAIs. Example of a broad class of intervention includes environmental cleaning. Environmental cleaning is aimed at removing or inactivating pathogens in the environment [20]. Generally, hospital surface decontamination is performed by environmental health personnel, who manually apply liquid disinfectant to the surfaces on a regular basis. Another method of environmental cleaning is surface wiping. This can be done by anyone including healthcare workers by wiping a surface immediately after touching it. Surface wiping relies on the mechanical removal of contamination, thus, does not require a strong microbicidal formulation [21].

Generally, colonized or infected individuals are the sources of MRSA in the environment. Approximately 10$30 \%$ of the general population has $S$. aureus colonization on their skin or in their noses [22]. Studies conducted in the 1960 s revealed that $S$. aureus is not freely suspended in air or on surfaces, but rather carried by desquamated epithelial cells $[23,24]$. As many as $10^{6}$ to $10^{7}$ cells, with dimension ranging from 8 to $20 \mu \mathrm{m}$, can be dispersed from the body in $24 \mathrm{~h}$. These aerial epithelial cells settle onto surfaces, but they may become temporarily reaerosolized when they are mechanically disturbed, only to redeposit back to the surfaces [25]. Huang et al found that patients, admitted to rooms previously occupied by MRSA-positive patients, had increased odds of subsequent MRSA acquisition [26]. However, the mechanisms describing how shedding from one patient leads to exposure and acquisition in another are yet to be well characterized. Possible exposure pathways include direct skin-to-skin contact and indirect environmental spread via contaminated environmental surfaces.

Healthcare workers (HCWs) play important roles in the transmission of healthcare-associated pathogens such as MRSA. HCWs colonized with MRSA, although rarely reported, have been shown to be important sources of infections that have led to outbreaks [17]. Additionally, HCWs have been implicated as mechanical vectors of transmission between patients [17]. A few modeling studies examined the importance of HCWs as vectors $[27,28]$, but did not explicitly include the environment. Other models that explicitly incorporate the environment have examined control strategies and the key interactions among patients, HCWs, and their environment [29-31]. Considering these studies, we have focused on a more comprehensive exposure model that explicitly accounts for fate and transport processes and provides a platform to investigate the effects of environmental interventions.

Specifically, we developed an exposure assessment model where MRSA is continuously shed from a colonized patient into the environment and is spread through HCWs' hands acting as vectors to another patient. The model includes one colonized patient, one uncolonized patient, and HCWs who care for them. The analysis was focused on MRSA exposure pathways to HCWs and the uncolonized patient. Direct MRSA exposure to the HCW was quantified by the net flow of MRSA resulting from the skin-to-skin contact with the colonized patient, while indirect exposure was quantified by the net flow of MRSA due to contamination of two surface types in the room, porous and nonporous. We employed similar procedures to quantify both direct as well as indirect exposure to the uncolonized patient.

\section{Methods}

\section{Model overview}

We constructed and analyzed a deterministic compartmental model of MRSA fate and transport between two hypothetical hospital rooms. In one room, there is a colonized patient, who is the only MRSA source in the system. A colonized patient is defined as an individual who harbors MRSA on the skin and in the nose, both of which produces a constant level of MRSA. This colonized patient sheds MRSA onto environmental surfaces both by: (1) aerial dispersal of MRSA-contaminated desquamated epithelial cells that eventually get deposited onto a surface; and (2) the touching of the surface with contaminated hands. HCWs visit the colonized patient's room hourly. While in the room, HCW's hands become contaminated by touching these contaminated surfaces and by contact with the colonized patient.

In the second room, there is an uncolonized patient that may become exposed either by touching contaminated surfaces or by contact with HCWs. The HCW's contaminated hands are the only sources of MRSA in this room. HCWs were not colonized with MRSA and do not shed MRSA; instead, they served as vectors of the transmission process. We assume that each $\mathrm{HCW}$ works an eight-hour shift. Each hour of a shift is organized identically as follows: The HCW visits the colonized patient's room during the first 20 minutes, the uncolonized patient's room during the next 20 minutes, and the HCWs' station during the last 20 minutes. At the HCW's station, we assume that there is no surface touching, thus, no MRSA transfer. This cycle is repeated every hour throughout the eight-hour shift. During each room visit, the HCW touches the patient and the two environmental surfaces at specified touching rates $\left(\tau_{\mathrm{hcw}-\mathrm{pt}}\right.$ and $\tau_{\text {hcw-sf }}$, respectively). Patients also touch the surfaces at a specified touching rate $\left(\tau_{\mathrm{pt}-\mathrm{sf}}\right)$. 
Our model is an ordinary differential equation-based model consisting of 10 compartments as shown in Figure 1. These compartments track the number of pathogens in the following locations: (1) on the colonized patient's skin, (2) on the uncolonized patient's skin, (3) on the HCW's skin, (4) on the porous surfaces in the colonized patient's room, (5) on the porous surfaces in the uncolonized patient's room, (6) on the nonporous surfaces in the colonized patient's room, (7) on the nonporous surfaces in the uncolonized patient's room, (8) in the uncolonized patient's nose, (9) in the HCW's nose, and (10) in the colonized patient's nose. The nose of a colonized patient, one of the sources of MRSA, is assumed to be at steady state with constant MRSA contamination levels. However, the uncolonized patient and the uncolonized HCWs did not have MRSA in their noses at the start of the simulation; their noses became contaminated with MRSA once their hands touch the noses. Each compartment representing presence of MRSA on the skin of either patients or HCWs is composed of two components, namely, "hand-skin" and "exposed non-hand-skin," and those are homogeneously mixed. Touching rates and transfer efficacies are associated only with the hand-skin, a proportional component of the skin compartment. MRSA concentration within each compartment is assumed to be instantaneously and homogenously spread.

\section{Model description}

MRSA shedding to the environment upon admission of a colonized patient occurs through two pathways: (1) continuous MRSA dispersal into the air on desquamated epithelial cells $(\alpha)$ and (2) intermittent MRSA transfer from the colonized patient's nose to hands $\left(\rho_{n}\right)$ to environmental surfaces $\left(\rho_{p}, \rho_{\text {np }}\right)$. MRSA in the air is assumed to be instantaneously settled onto environmental surfaces. Once transferred onto environmental surfaces (or skin and hands), some MRSA might naturally die off $\left(\mu_{\mathrm{sk}}, \mu_{\mathrm{p}}, \mu_{\mathrm{np}}\right)$. When HCWs and patients touch these contaminated surfaces, some fraction of MRSA might be picked up. This fraction depends on the transfer efficiency

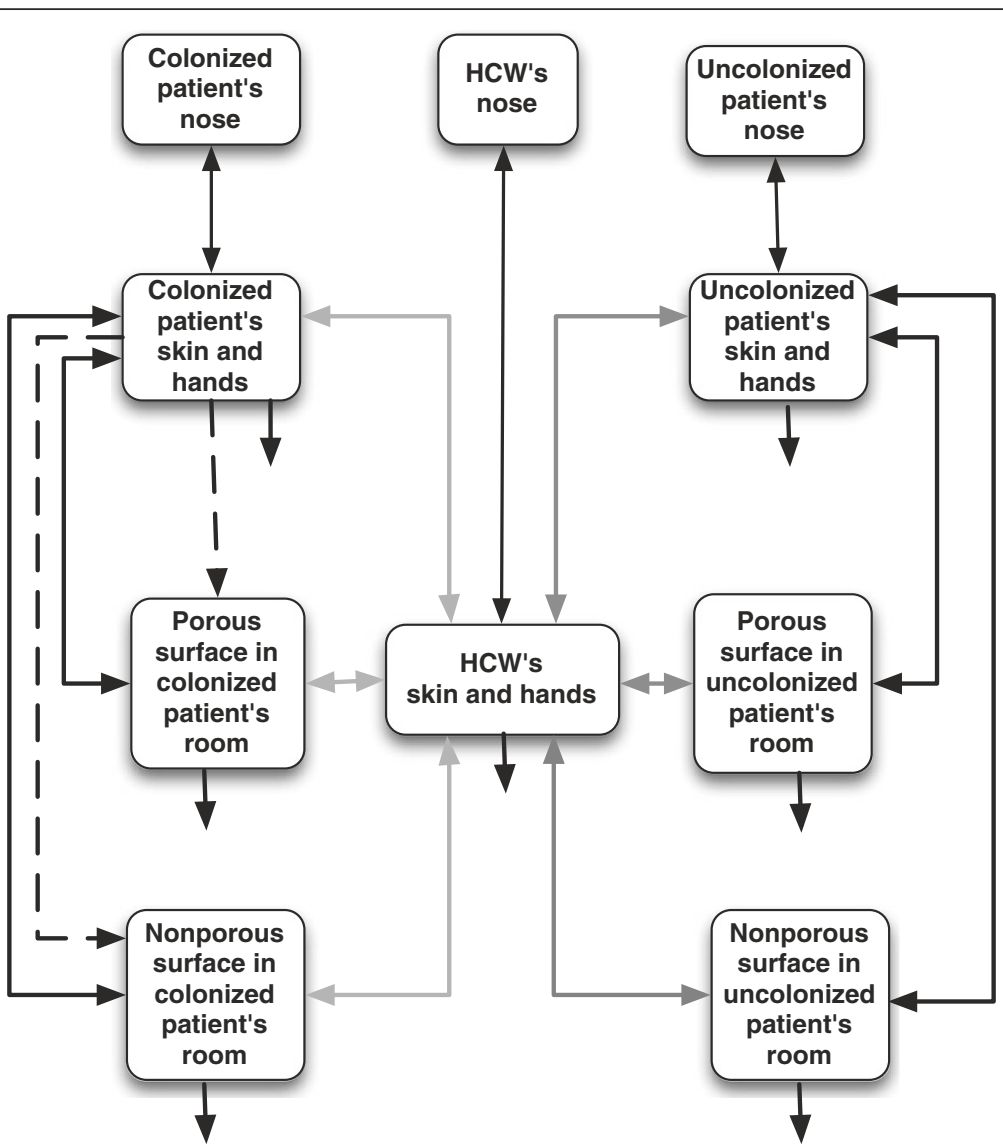

Figure 1 Diagram of our 10 compartments fate and transport exposure model. Solid arrows represent pathogen flows due to touching events or natural die off. Dashed arrows represent shedding from colonized patient to the porous and nonporous surfaces in the room. Black arrows represent time independent flows within the colonized and uncolonized patient's room, and touching noses. Grey arrows represent time dependent flows to and from HCW's skin and hands during the first 20 minutes of the hour (light grey) and during the next 20 minutes of the hour (dark grey). 
$\left(\rho_{\mathrm{p}}, \rho_{\mathrm{np}}\right)$ and the MRSA quantity on the surfaces and skin. Once MRSA is transferred onto the skin of either patients or HCWs, it might be redeposited back to surfaces $\left(\rho_{\mathrm{p}}, \rho_{\mathrm{np}}\right)$. Finally, MRSA on the skin might be inoculated to the nose, when individuals touch their noses with their contaminated hands.

Table 1 presents model parameters. The Additional file 1 provides the justification for the parameter values used in our model, while the Additional file 2 provides the equations used in the model.

\section{Surface touching event}

Surface touching is the event that transfers MRSA from one compartment to another. For each touching event,

Table 1 Model parameters and their values

\begin{tabular}{|c|c|c|}
\hline & Parameter & Value \\
\hline \multicolumn{3}{|l|}{ Shedding parameters: } \\
\hline Shedding (air dispersal) rate (cfu/ $\left.\mathrm{cm}^{2} / \mathrm{min}\right)$ & a & 0.01 \\
\hline \multicolumn{3}{|l|}{ Survival parameters: } \\
\hline Die-off rate on skin and hand $\left(\mathrm{min}^{-1}\right)$ & $\mu_{\mathrm{sk}}$ & 0.00353 \\
\hline Die-off rate on porous surface $\left(\mathrm{min}^{-1}\right)$ & $\mu_{p}$ & 0.000632 \\
\hline Die-off rate on nonporous surface $\left(\mathrm{min}^{-1}\right)$ & $\mu_{\mathrm{np}}$ & 0.0002 \\
\hline \multicolumn{3}{|l|}{ Contact parameters: } \\
\hline Rate of patient touching surfaces $\left(\mathrm{min}^{-1}\right)$ & $T_{p t-s f}$ & 0.134 \\
\hline Rate of HCW touching patient $\left(\mathrm{min}^{-1}\right)$ & $T_{\text {hcw-pt }}$ & 0.4 \\
\hline Rate of HCW touching surfaces $\left(\mathrm{min}^{-1}\right)$ & $T_{\text {hcw-sf }}$ & 0.4 \\
\hline Rate of touching the nose $\left(\mathrm{min}^{-1}\right)$ & $\tau_{n}$ & 0.025 \\
\hline $\begin{array}{l}\text { Rate of HCW wiping nonporous } \\
\text { surface }\left(\min ^{-1}\right)\end{array}$ & $\omega_{\mathrm{np}}$ & 0.4 \\
\hline \multicolumn{3}{|l|}{ Transfer efficiency parameters: } \\
\hline $\begin{array}{l}\text { Transfer efficiency from porous surface } \\
\text { to hand }\end{array}$ & $\rho_{p}$ & 0.1 \\
\hline $\begin{array}{l}\text { Transfer efficiency from nonporous } \\
\text { surface to hand }\end{array}$ & $\rho_{\mathrm{np}}$ & 0.4 \\
\hline Transfer efficiency from hand to skin & $\rho_{\mathrm{sk}}$ & 0.35 \\
\hline Transfer efficiency from fingertip to nose & $\rho_{\mathrm{n}}$ & 0.2 \\
\hline \multicolumn{3}{|l|}{ Surface area parameters: } \\
\hline $\begin{array}{l}\text { Total exposed skin and hand surface } \\
\text { area of patients }\left(\mathrm{cm}^{2}\right)\end{array}$ & $A_{p t}$ & 2000 \\
\hline $\begin{array}{l}\text { Total exposed skin and hand surface } \\
\text { area of HCWs }\left(\mathrm{cm}^{2}\right)\end{array}$ & $A_{\text {how }}$ & 2000 \\
\hline Total porous surface area $\left(\mathrm{cm}^{2}\right)$ & $A_{p}$ & 2000 \\
\hline Total nonporous surface area $\left(\mathrm{cm}^{2}\right)$ & $A_{n p}$ & 2000 \\
\hline Nose surface area $\left(\mathrm{cm}^{2}\right)$ & $A_{n}$ & 4 \\
\hline Hand contact surface area $\left(\mathrm{cm}^{2}\right)$ & $A_{h}$ & 150 \\
\hline Fingertip contact surface area $\left(\mathrm{cm}^{2}\right)$ & $A_{f}$ & 1 \\
\hline \multicolumn{3}{|l|}{ Interventions: } \\
\hline Daily surface decontamination efficacy & $\varepsilon_{\mathrm{d}}$ & $0,50,100 \%$ \\
\hline Wiping efficacy & $\varepsilon_{w}$ & $0,50,100 \%$ \\
\hline
\end{tabular}

there are bidirectional flows of MRSA to and from the two contacting surfaces. The net quantity of these two bidirectional flows determines whether a contacting surface will be the net-giver or the net-receiver, thus determining when the resultant event is the contamination of the touched surface or exposure to the patients/HCWs.

In this model, we compared two exposure pathways in the two patient's rooms: (1) direct and indirect exposure to the HCWs in the colonized patient's room, and (2) direct and indirect exposure to the uncolonized patient's room. Direct exposure resulted from the skin-to-skin contact between the HCWs and the patients. Indirect exposure resulted from the skin-tosurface-to-skin contact.

In addition to the direct and indirect exposures, HCWs and patients touched their noses with their fingertips. For the colonized patient, this type of contact led to hand contamination, which later contaminated surfaces when the patient touched the surfaces. For the uncolonized patient and the HCWs, touching noses resulted in increase in MRSA concentration in their noses, but did not lead to changes in the colonization status.

To illustrate the contact-mediation processes, we considered an example of a direct contact event where a $\mathrm{HCW}$ touched the uncolonized patient (Additional file 2: Table S2). For each touch, a quantity of MRSA was transferred from the HCW's hand to the patient (HCW*150/ $\left.2000^{*} \rho_{\mathrm{sk}}\right)$ as well as from the patient to the HCW's hand $\left(\mathrm{PT}_{\mathrm{u}}{ }^{*} 150 / 2000 * \rho_{\mathrm{sk}}\right)$. The transferred MRSA quantities depend on the following: (1) the bacterial concentrations at both contacting surfaces (i.e., $\mathrm{HCW}$ and $\mathrm{PT}_{\mathrm{u}}$ ), (2) the contact surface area (i.e., 150 sq.cm.), (3) the total surface area (i.e., 2000 sq.cm.), and (4) transfer efficiency. We assumed a symmetrical transfer efficiency, which implied that the fraction of pathogen that was transferred from the HCW to the uncolonized patient was the same as the fraction transferred from the uncolonized patient to the $\mathrm{HCW}$. This fraction is the transfer efficiency of hands to skin or skin to hands $\left(\rho_{\text {sk }}\right)$. The net quantity of pathogens transferred depends on the contamination levels on contacting surfaces. In this case, HCWs were the only sources of MRSA in the uncolonized patient's room. Thus, the direct contact of HCWs and the uncolonized patient resulted in an increase in contamination of the uncolonized patient, i.e., direct exposure to the uncolonized patient.

Indirect contact involved the contamination of surfaces. When a surface was touched, the contamination from the fingers was assumed to be spread out equally throughout the surface, i.e., the surface was considered spatially homogeneous.

\section{Model interventions}

Our analysis focused on the effect of two environmental cleaning interventions: surface decontamination and 
surface wiping after touching. Surface decontamination was performed on the entire areas of both porous and nonporous surfaces, once every $24 \mathrm{~h}$. Following each decontamination event, a fraction of MRSA was removed depending on the cleaning efficacy of surface decontamination $\left(\varepsilon_{\mathrm{d}}\right)$.

Surface wiping was only performed on nonporous surfaces, merely on a fraction of the surface corresponding to the size of the HCW's hand. After touching a nonporous surface, the HCWs wiped the surface. Thus, the wiping rate $\left(\omega_{\mathrm{np}}\right)$ was identical to the rate at which HCWs touched the nonporous surfaces $\left(\tau_{\mathrm{hcw} \text {-sf }}\right)$. Following each wipe, a fraction of MRSA was removed depending on the cleaning efficacy of wiping $\left(\varepsilon_{\mathrm{w}}\right)$. Because of the homogeneous nature of the surface, the HCWs wiped a random part of the surface, not necessarily where touching occurred.

\section{Model analyses}

Our analysis is divided into two parts. First, we describe the MRSA contamination levels on all surfaces and skin in the absence of any intervention. We compare the relative importance of direct versus indirect exposure to the HCWs and the uncolonized patient. Direct exposure to the HCW was quantified by the net flow of MRSA resulting from the skin-to-skin contact with the colonized patient, while indirect exposure to the HCW was quantified by the net flow of MRSA from touching the two surfaces in the room. Analogous procedures were used to quantify direct and indirect exposure to the uncolonized patient.

Second, we evaluate and compare the effect of the two interventions: surface decontamination and surface wiping. Surface decontamination and wiping were both evaluated with $50 \%$ and $100 \%$ cleaning efficacy.

\section{Sensitivity analyses}

We explored the sensitivity of our findings with respect to varying values of the transfer efficiency, die-off rates, and contact rates. The results, presented in the Additional file 3 , suggest that our conclusions are robust to parameter uncertainty and variability.

\section{Results}

The dynamics of each compartment are shown in Figure 2. MRSA, both on surfaces and skin of patients, quickly came to a steady state. Based on the parameter values shown in Table 1, steady state was reached following a perturbation in approximately $24 \mathrm{~h}$. The relative steady state values can be explained by the fact that the colonized patient was the only source of MRSA; higher MRSA levels found in the room occupied by the colonized patient. Due to the fact that surfaces have slower MRSA die-off rates than the skin, we found higher MRSA levels on surfaces compared to skin. These higher contamination levels on the surfaces reflect the importance of the indirect route (patient to surfaces to $\mathrm{HCW}$ ) in the colonized patient's room. In our simulations, $70 \%$ of the contamination of the HCW, while in the colonized patient's room, came through this indirect exposure route. Of this, $66 \%$ resulted from touching the nonporous surface rather than the porous surface. We found the situation in the uncolonized patient's room was just reversed; $65 \%$ of the contamination of the uncolonized patient came through direct exposure, when the HCW touched the patient.

The relative levels of MRSA contamination on each surface type were reversed in each room (Figure 2). In the colonized patient's room, the porous surface had higher MRSA contamination, while in the uncolonized patient's room, the nonporous surface had higher MRSA contamination. This difference occurred despite the equal distribution of the aerosolized shedding on both the porous and nonporous surfaces in the colonized patient's room. This was because the nonporous surface had a higher transfer efficiency; therefore, transferred more MRSA to the HCW when touched. Thus, the nonporous fomite was more important in facilitating the spread of MRSA, despite having a lower steadystate MRSA contamination level. The porous surface, with the higher MRSA levels, can be thought of as a sentinel fomite. In the uncolonized patient's room, with no host shedding, the nonporous surface received more contamination due to its higher transfer efficiency; thus, higher MRSA contamination levels would be expected on the nonporous surface compared to the porous surface.

\section{Surface decontamination}

Following decontamination on all surfaces, there was immediate recontamination. Within $24 \mathrm{~h}$, MRSA contamination returned to the pre-decontamination levels (Figure 3). Frequent surface decontamination, therefore, resulted in a more sustained decrease in MRSA contamination.

These effects trickled throughout the system, as evidenced by the decreased MRSA concentrations on the skin of the colonized patient, uncolonized patient, and HCW, despite not being cleaned directly. With 50\% cleaning efficacy, the total exposure to the uncolonized patient was reduced by $15 \%$, while with $100 \%$ cleaning efficacy, the exposure was reduced by $30 \%$ (Figure 4).

\section{Surface wiping}

With surface wiping, there was no jagged pattern, as seen with daily decontamination; instead, there was a cyclical pattern due to the HCW visits to patient rooms (Figure 5). Surface wiping with 50\% cleaning efficacy reduced the total exposure to the uncolonized patient 


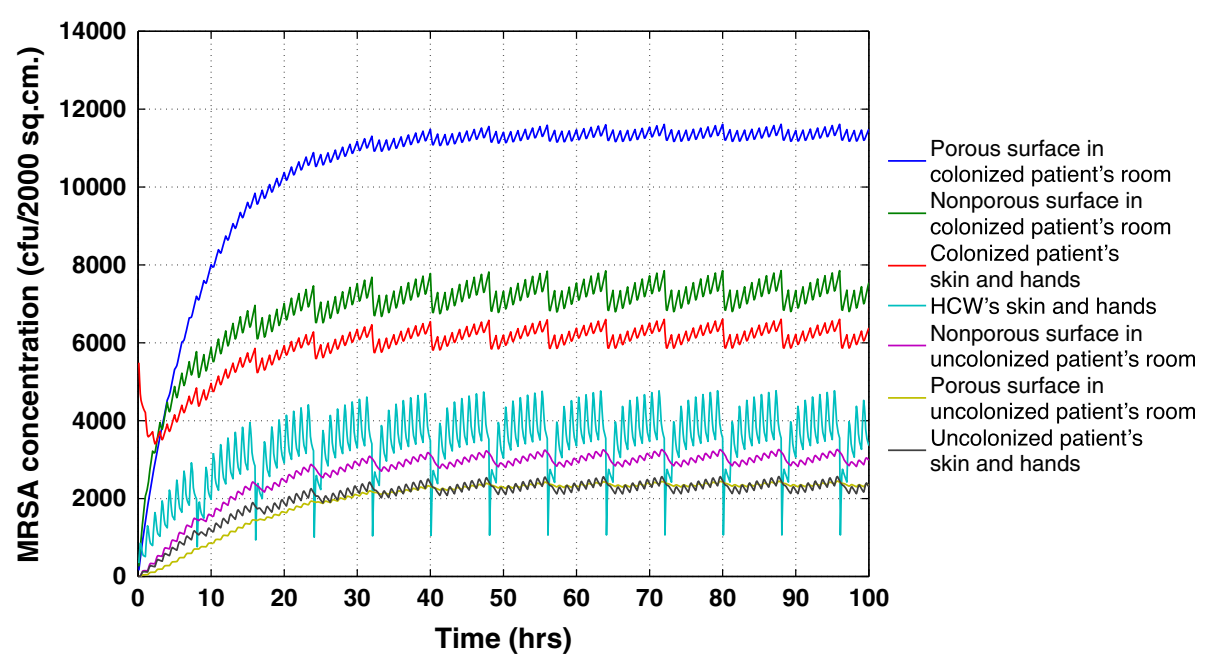

Figure 2 MRSA concentrations at baseline scenario without intervention. The Y-axis represents MRSA concentrations on the entire surface area $\left(\mathrm{cfu} / 2000 \mathrm{~cm}^{2}\right)$ from the seven compartments. These compartments starting with the highest MRSA concentrations, are: 1) the porous surface in the colonized patient's room; 2) the nonporous surface in the colonized patient's room; 3) the exposed skin and hands of the colonized patient; 4) the exposed skin and hands of the HCW; 5) the nonporous surface in the uncolonized patient's room; 6) the porous surface in the uncolonized patient's room; and 7) the exposed skin and hands of the uncolonized patient.

substantially (52\% reduction in exposure), while with $100 \%$ cleaning efficacy, the increased effect was less than linear (65\% reduction in exposure). Because surface wiping was effectively targeted as intervention against MRSA on nonporous surfaces, the nonporous surfaces were affected more by wiping than porous surfaces; in particular, the nonporous surface in the uncolonized patient's room had the highest reduction $(81 \%$, given a $100 \%$ cleaning efficacy) (Figure 6).

\section{Comparison of surface decontamination and surface wiping}

The efficacy of surface decontamination and surface wiping are both depend on the following factors: (1) total surface area cleaned per day, (2) cleaning frequency, (3) proportion of surface area cleaned at each implementation, (4) type of surface cleaned, (5) cleaning efficacy, and (6) time of cleaning. Surface decontamination was assumed to clean the whole surface one to three times daily,

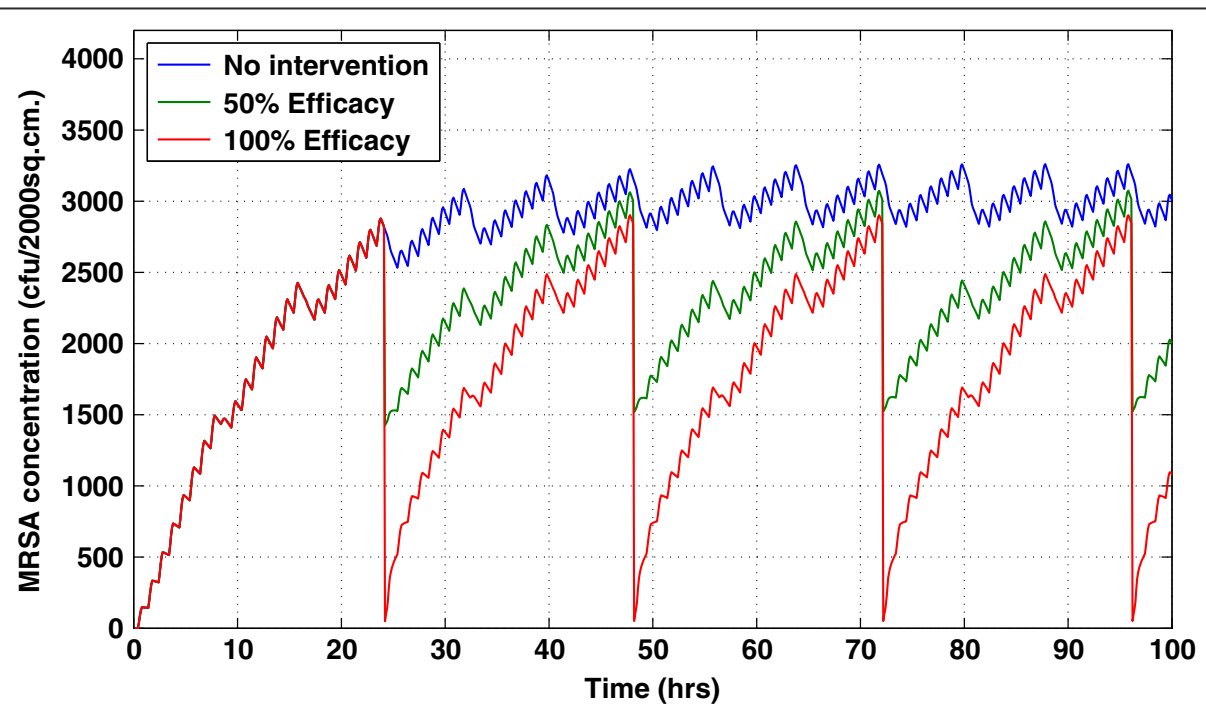

Figure 3 Temporal effects of daily surface decontamination on the nonporous surface in the uncolonized patient's room using $50 \%$ and $100 \%$ efficacy compared to when there is no intervention. Surface decontamination was performed on both porous and nonporous surfaces. Due to the continuous shedding of the colonized patient, the effect of daily decontamination was short-lived; this pattern is consistently observed in other compartments. 


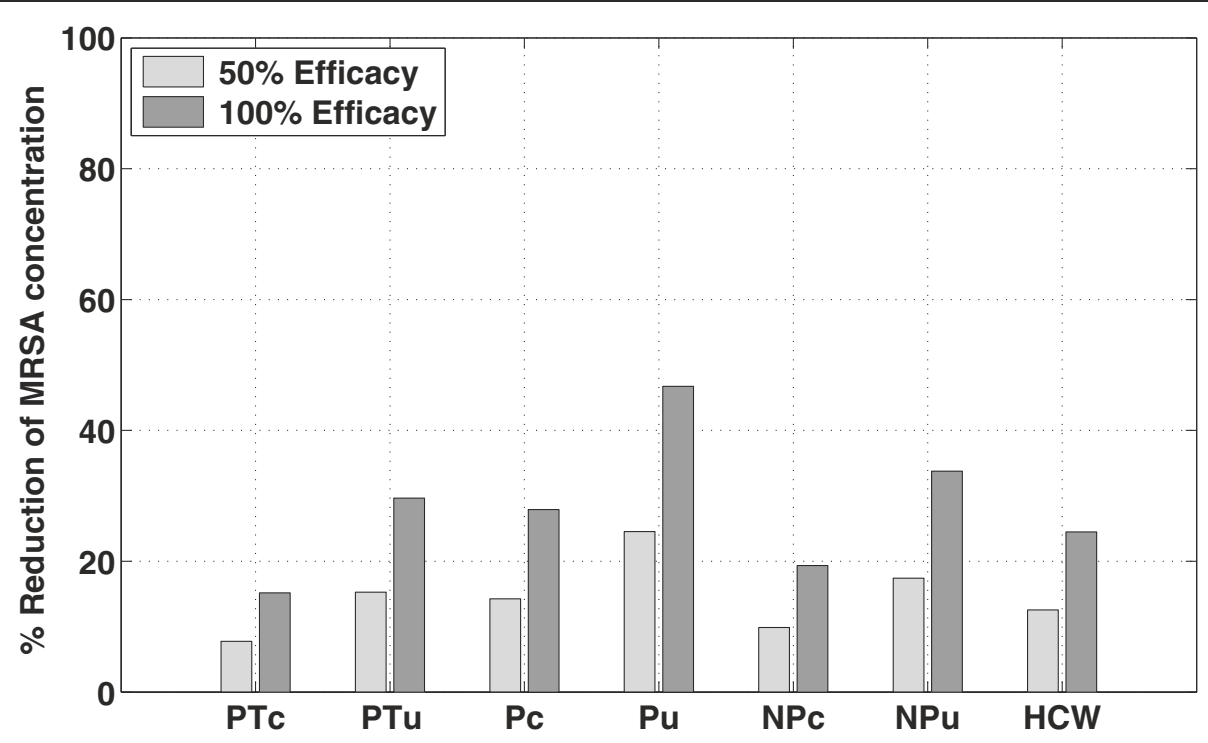

Figure 4 MRSA reduction in seven compartments due to daily surface decontamination. The $Y$-axis is the percentage reduction of MRSA concentration using 50\% and 100\% efficacy compared to when there is no intervention. The seven compartments are the exposed skin and hands of the colonized patient (PTc), the exposed skin and hands of the uncolonized patient (PTu), the porous surface in the colonized patient's room $(\mathrm{Pc})$, the porous surface in the uncolonized patient's room $(\mathrm{Pu})$, the nonporous surface in the colonized patient's room (NPc), the nonporous surface in the uncolonized patient's room (NPu), and the exposed skin and hands of the HCW. The porous surface in the uncolonized patient's room had the highest reduction (46\% for 100\% efficacy).

whereas surface wiping cleaned a random proportion of the surface $\left(150 \mathrm{~cm}^{2} / 2000 \mathrm{~cm}^{2}\right)$ eight times per hour. To compare the two intervention approaches, cleaning efficacy was held constant, at $100 \%$. Surface decontamination always starts at time zero, and HCWs wiped after every touch, unless specified differently. Following surface touching, MRSA contamination was spread out equally throughout the surface. We made a similar assumption that the HCW wiped a random portion of the surface, not necessarily where touching occurred.

We varied the total surface area cleaned per day by varying the cleaning frequency and surface area cleaned at each implementation (Table 2). Daily surface decontamination affected a large surface area at each implementation (four surfaces, each with $2000 \mathrm{~cm}^{2}$ ), while surface wiping affected a much smaller surface area

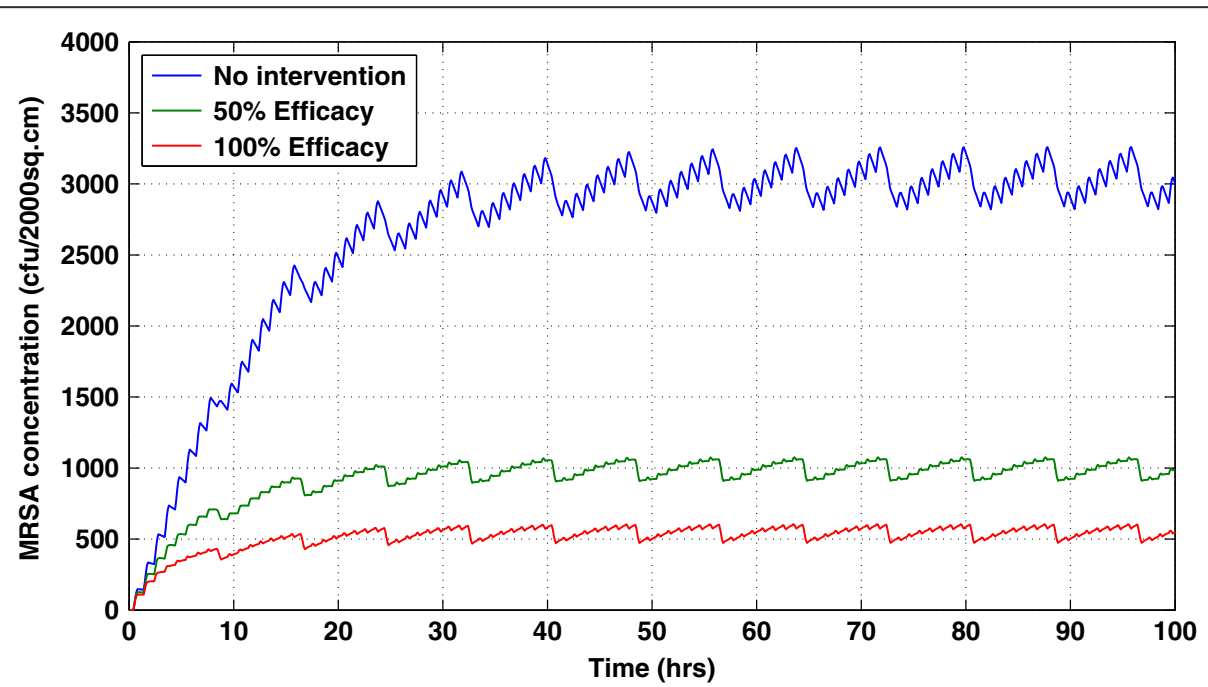

Figure 5 Temporal effects of surface wiping on the nonporous surface in the uncolonized patient's room using $50 \%$ and $100 \%$ efficacy compared to when there is no intervention. Surface wiping only occurs on nonporous surfaces. Surface wiping consistently suppresses MRSA concentration levels; small fluctuations are due to HCW's visit to patient's room every 8-hour. 


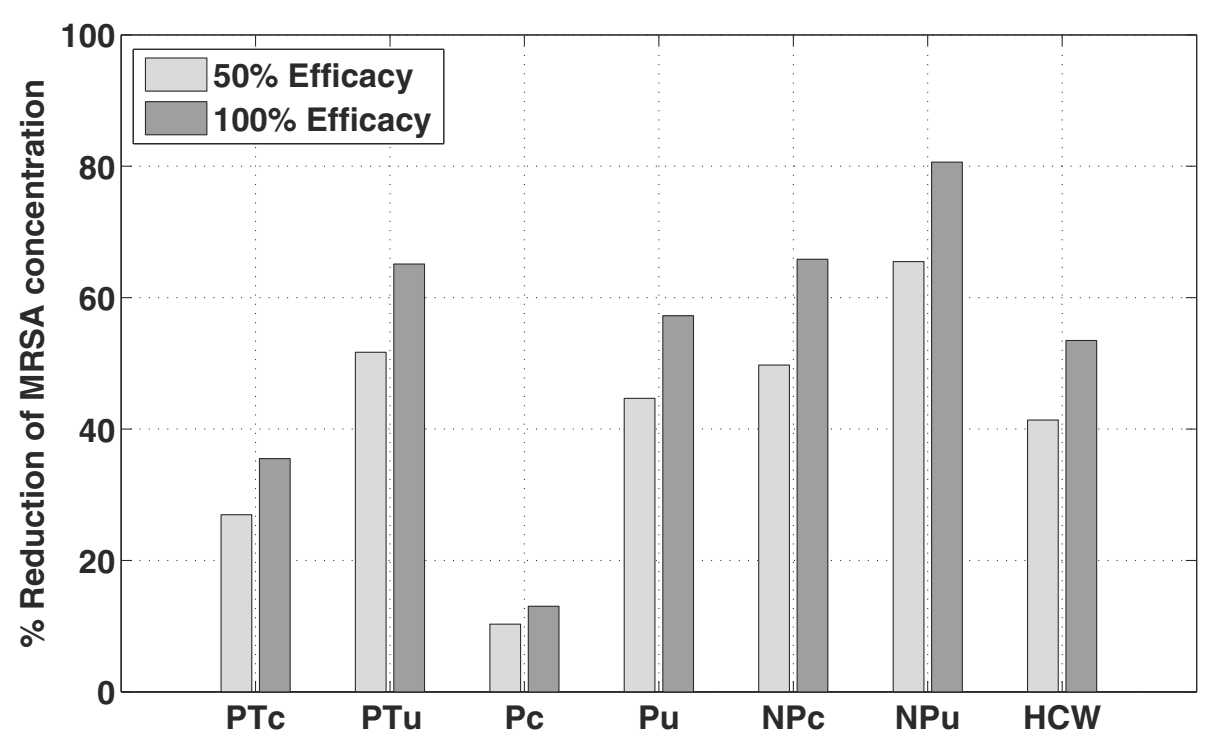

Figure 6 MRSA reduction in seven compartments due to surface wiping. The Y-axis is the percentage reduction of MRSA concentration from surface wiping using 50\% and 100\% efficacy compared to when there is no intervention. The seven compartments are the exposed skin and hands of the colonized patient (PTc), the exposed skin and hands of the uncolonized patient (PTu), the porous surface in the colonized patient's room $(\mathrm{Pc})$, the porous surface in the uncolonized patient's room (Pu), the nonporous surface in the colonized patient's room (NPc), the nonporous surface in the uncolonized patient's room (NPu), and the exposed skin and hands of the HCW. The nonporous surface in the uncolonized patient's room had the highest reduction ( $81 \%$ for $100 \%$ efficacy).

$\left(150 \mathrm{~cm}^{2}\right)$ with each wipe. However, because the cleaning frequency of wiping was many times per hour, wiping cleaned a much larger total surface area per day $\left(57600 \mathrm{~cm}^{2} /\right.$ day vs. $8000 \mathrm{~cm}^{2} /$ day).

When surface decontamination was performed daily (Table 2 scenario A) and surface wiping was performed after each surface touch by HCWs (Table 2 scenario D), we found wiping was more effective. When surface decontamination was performed once every eight hours (Table 2 scenario $\mathrm{C}$ ), the uncolonized patient had similar levels of MRSA exposure, despite the fact that surfacedecontamination cleaned less than half the surface area that surface wiping could clean. We found that the surface decontamination performed better than surface wiping, when the two interventions were adjusted to clean the same total daily surface area (Table 2 scenarios $\mathrm{C}$ and $\mathrm{E}$ ). However, this was an artifact that the $\mathrm{HCW}$ did not clean the specific area that they contaminated. To address this, incorporating spatial heterogeneity in the modeling of the surfaces is required.

\section{Discussion}

Our model for MRSA exposure assessment highlights the dynamic interplay between MRSA colonized and uncolonized patients, HCWs, and environmental surfaces. Environmental cleaning has been under-appreciated; therefore, underutilized as an intervention option in the hospital setting. The analysis presented here highlights the importance of the indirect exposure route, which suggests the potential of environmental cleaning

Table 2 Intervention effectiveness when total surface area cleaned per day is varied

\begin{tabular}{|c|c|c|c|c|c|c|}
\hline \multirow[t]{2}{*}{ Cleaning frequency } & \multirow{2}{*}{$\begin{array}{c}\text { No } \\
\text { Intervention }\end{array}$} & \multicolumn{3}{|c|}{ Surface decontamination } & \multicolumn{2}{|c|}{ Surface wiping } \\
\hline & & A. Every $24 \mathrm{~h}$ & B. Every 12 h & C. Every $8 \mathrm{~h}$ & $\begin{array}{l}\text { D. HCW swipe } \\
8 \text { times/h }\end{array}$ & $\begin{array}{l}\text { E. HCW swipe } \\
3 \text { times/h* }\end{array}$ \\
\hline Total surface area cleaned/day $\left(\mathrm{cm}^{2}\right)$ & & 8000 & 16000 & 24000 & 57600 & 24000 \\
\hline Types of surfaces & & Both & Both & Both & Nonporous & Nonporous \\
\hline Number of cleaning events/day & & 1 & 2 & 3 & 192 & 80 \\
\hline Surface area cleaned each implementation & & 2000 & 2000 & 2000 & 150 & 150 \\
\hline $\begin{array}{l}\text { Average MRSA concentration on the } \\
\text { uncolonized patient }\end{array}$ & 2065.55 & 1454.76 & 1055.09 & 776.87 & 703.89 & 1111.86 \\
\hline $\begin{array}{l}\text { Percent reduction of exposure to the } \\
\text { uncolonized patient }\end{array}$ & $\mathrm{n} / \mathrm{a}$ & $29.57 \%$ & $48.92 \%$ & $62.38 \%$ & $65.92 \%$ & $46.17 \%$ \\
\hline
\end{tabular}

*HCWs touch the nonporous surface 8 times an hour and wipe only 3 times an hour. 
of surfaces as an integral component of MRSA infection control.

Specifically, our analysis suggests that environmental surfaces are key exposure sources that contaminate the hands. A randomized crossover study on the impact of enhanced cleaning in intensive care units (ICUs) supports our findings that enhanced cleaning of highcontact surfaces not only significantly reduced the number of MRSA isolates in the environment but also on the hands of the staff [32]. These conclusions remained, even when HCWs touched patients as often as they touched surfaces, largely due to the higher die-off rates on hands and skin compared to surfaces. In reality, HCWs touch room surfaces more frequently than they touch patients [33], indicating that we might be underestimating the importance of indirect exposure through environmental surfaces.

HCWs are frequently viewed as vectors of transmission, also assumed in our model $[17,19]$. A study of an isolation ward for six epidemic MRSA (EMRSA) patients provides strong evidence that the transient and shortterm carriage in HCWs is not uncommon and probably results in the transfer of EMRSA between patients [34]. However, HCWs may also be the source of transmission [17]. A six-year surveillance of an operating room technician, who was colonized with S. aureus, demonstrated transmission from this technician to patients in a number of situations [35]. Since our model only considered the HCWs as mechanical vectors, we probably underestimated the exposure to the uncolonized patient.

Given the role of HCW's hands in spreading contamination, hand hygiene is potentially a strong effect modifier of cleaning. This is clearly true if hand hygiene is done perfectly, i.e., if contamination never gets on the hand, surface cleaning is irrelevant. In reality, however, hand hygiene is never perfectly implemented. Real-world impediments include the following: (1) imperfect compliance with hand hygiene procedures [36], (2) crosscontamination of $\mathrm{HCW}$ hands when HCWs touch their own contaminated clothing $[16,18]$, and (3) recontamination due to the touching of contaminated surfaces [15]. Because of these complications, we chose not to include hand hygiene in this analysis as it would have required a more sophisticated model, which would incorporate spatial heterogeneity of the skin, i.e., differentiating between the hand and other parts of the body. Although beyond the scope of this manuscript, describing the joint effects of hand hygiene (considering compliance and recontamination) and surface cleaning would provide valuable information for developing realistic intervention strategies.

Because of these limitations, implementing a hand hygiene intervention in conjunction with other interventions is necessary to decrease MRSA transfer and exposure to the uncolonized patients. Surface decontamination cleans thoroughly entire surfaces, when implemented, but it cannot feasibly do so very often. Surface wiping is less thorough, only cleans very small segments of surfaces, but does so quite often. Despite the lack of thoroughness, we found that surface wiping was more effective than daily surface decontamination as the former cleaned more surface area per day. Furthermore, our assumption that surfaces were homogeneous underestimated the importance of wiping, where wiping generally cleaned areas that had been touched rather than cleaning the surface proportionally, in an untargeted manner.

While new technology that provides high levels of disinfection (e.g., hydrogen peroxide vapor) has been shown to effectively eliminate bacteria from the environment $[37,38]$, our analysis showed diminishing returns with increasing cleaning efficacy due to recontamination. Consistent with our finding, a prior study demonstrated that after eliminating MRSA from the environment by applying hydrogen peroxide vapor, recontamination occurred 24 hours after colonized patients were readmitted into the intensive care unit [37]. Further simulations suggested that by increasing the frequency of cleaning, additional reductions in the exposure to the uncolonized patient were achievable; i.e., increasing cleaning frequency was more important than increasing cleaning efficacy.

Our model assumed that surface decontamination thoroughly cleaned the entire surface. In reality, such thoroughness is often lacking. A review of environmental hygiene in healthcare settings showed that implementation often lacked thoroughness; only $40 \%$ of the surfaces were being thoroughly cleaned in accordance with existing policies [39]. Some have used a monitoring system to improve cleaning thoroughness, which resulted in decreased MRSA infection [40,41]. However, this approach often requires an extra person; for example, when hand-touchedsurfaces near patients and HCWs' station were cleaned more frequently by extra personnel, there was a $32.5 \%$ reduction in environmental contamination sites and 26.6\% reduction in new MRSA infections [41].

Similarly, our model assumed that surface wiping was conducted using proper techniques. The true effectiveness of surface wiping was difficult to define due to a wide variety of commercially available wipes and microfiber-based fabrics, as well as a variation in test protocols [21,42-47]. One study showed that wiping plastic surfaces three or more times with saline-moistened wipes is as effective as disinfectant wipes [45]. At the same time, disinfectant wipes may serve as vectors in transferring pathogen between surfaces when reused without proper cleaning [46,47]. Nevertheless, as suggested in our analysis, the frequency of wiping with presumably good technique can significantly reduce the environmental contamination. 
In addition, our findings are helpful in interpreting environmental surveillance data. When a MRSA-positive patient has recently been in a hospital room, our model will predict a higher concentration of MRSA on porous surfaces (e.g., bed sheets, mattresses, and blankets), all else being equal between the surfaces. On the other hand, for a MRSA-negative patient, our model will predict a higher concentration of MRSA on nonporous surfaces. This suggests the possibility that porous surfaces can act as a sentinel fomite indicating the recent presence of a MRSA-positive patient. Further analysis and data are required to support this finding.

\section{Conclusions}

In this study, we modeled only fate and transport, making no prediction on infection risk or any other interpretation of the total exposure dose to the uncolonized patient. According to the quantitative risk assessment paradigm, exposure assessment is an initial and essential step toward improving our understanding in transmission system. To develop a full environmental infection transmission system, a model requires a dose-response function in the mass action formulation. Our dynamic fate and transport modeling framework highlights that developing optimal control strategies depends on a number of environmental factors such as pathogen die-off rates on surfaces and hands, the transfer efficiency of pathogen movement from hand to surface and surface to hand, and a host of behavioral features that include rates of touching surfaces as well as colonization sites such as the nose and the skin. Improved data on these processes along with more detailed models that contain site-specific features would provide guidance on not just the effectiveness of any particular control strategy, but also the joint effects of multiple intervention strategies.

\section{Additional files}

Additional file 1: Supplementary material I. Supporting Evidence for Environment-mediated Transmission and Model Parameterization.

Additional file 2: Supplementary material II. Table S2 and differential equations.

Additional file 3: Supplementary material III. Sensitivity analysis.

\section{Competing interests}

The authors declare that they have no competing interests.

\section{Authors' contributions}

NP designed the study, constructed the model, performed the analysis, and drafted the manuscript. IHS, JSK, and JNSE reviewed the model, and provided critical advice. NP wrote the manuscript with major contributions from JNSE and other authors. All authors have read and approved the final manuscript.

\section{Acknowledgements}

The authors would like to thank Dr. Carol Chenoweth, Dr. Betsy Foxman,

Dr. Duane Newton, and Dr. Rick Riolo for their guidance in the study design and manuscript preparation and Dr. Jijun Zhao and Dr. Anuj Mubayi for their technical assistance. The authors would also like to thank the reviewers for valuable and insightful suggestions that helped to improve the quality of the manuscript.

This research was supported from (i) the Center for Advancing Microbial Risk Assessment by the US Environmental Protection Agency Science to Achieve Results (STAR) Program and by the US Department of Homeland Security University Programs (Grant no. R83236201), and (ii) the U.S. NIH sponsored Interdisciplinary Training Program in Infectious Diseases by the Molecular and Clinical Epidemiology of Infectious Diseases (NIH T32 Al049816).

Received: 29 April 2013 Accepted: 11 December 2013

Published: 17 December 2013

\section{References}

1. Diekema DJ, Pfaller MA, Schmitz FJ, Smayevsky J, Bell J, Jones RN, Beach M: Survey of infections due to Staphylococcus species: frequency of occurrence and antimicrobial susceptibility of isolates collected in the United States, Canada, Latin America, Europe, and the Western Pacific region for the SENTRY Antimicrobial Surveillance Program, 1997-1999. Clin Infect Dis 2001, 32(Suppl 2):S114-132.

2. Jarvis WR, Schlosser J, Chinn RY, Tweeten S, Jackson M: National prevalence of methicillin-resistant Staphylococcus aureus in inpatients at US health care facilities, 2006. Am J Infect Control 2007, 35(10):631-637.

3. Tiemersma EW, Monnet DL, Bruinsma N, Skov R, Monen JC, Grundmann H: Staphylococcus aureus bacteremia. EurEmerg Infect Dis 2005, 11(11):1798-1799.

4. CDC: Management of Multidrug-Resistant Organisms in healthcare settings. In The Healthcare Infection Control Practice Advisory Committee. 2006

5. Calfee DP, Salgado CD, Classen D, Arias KM, Podgorny K, Anderson DJ, Burstin H, Coffin SE, Dubberke ER, Fraser V, et al: Strategies to prevent transmission of methicillin-resistant Staphylococcus aureus in acute care hospitals. Infect Cont Hosp Epid 2008, 29(Suppl 1):S62-80.

6. Boyce JM, Pittet D: Guideline for Hand Hygiene in Health-Care Settings: recommendations of the Healthcare Infection Control Practices Advisory Committee and the HICPAC/SHEA/APIC/IDSA Hand Hygiene Task Force. Infect Cont Hosp Epid 2002, 23(12 Suppl):S3-40.

7. Lee AS, Huttner B, Harbarth S: Control of methicillin-resistant Staphylococcus aureus. Infect Dis Clin North Am 2011, 25(1):155-179.

8. Muto CA, Jernigan JA, Ostrowsky BE, Richet HM, Jarvis WR, Boyce JM, Farr BM: SHEA guideline for preventing nosocomial transmission of multidrug-resistant strains of Staphylococcus aureus and enterococcus. Infect Control Hosp Epidemiol 2003, 24(5):362-386.

9. Pittet D, Boyce JM: Hand hygiene and patient care: pursuing the Semmelweis legacy. Lancet Infect Dis 2001, 1:9-20.

10. Mortimer EA Jr, Lipsitz PJ, Wolinsky E, Gonzaga AJ, Rammelkamp CH Jr: Transmission of staphylococci between newborns. Importance of the hands to personnel. Am J Dis Child 1962, 104:289-295.

11. Bolon M: Hand hygiene. Infect Dis Clin North Am 2011, 25(1):21-43.

12. Randle J, Arthur A, Vaughan N: Twenty-four-hour observational study of hospital hand hygiene compliance. J Hosp Infect 2010, 76(3):252-255.

13. Beggs CB, Shepherd SJ, Kerr KG: Increasing the frequency of hand washing by healthcare workers does not lead to commensurate reductions in staphylococcal infection in a hospital ward. BMC Infect Dis 2008, 8:114.

14. Beggs CB, Shepherd SJ, Kerr KG: How does healthcare worker hand hygiene behaviour impact upon the transmission of MRSA between patients?: an analysis using a Monte Carlo model. BMC Infect Dis 2009, 9:64.

15. Bhalla A, Pultz NJ, Gries DM, Ray AJ, Eckstein EC, Aron DC, Donskey CJ: Acquisition of nosocomial pathogens on hands after contact with environmental surfaces near hospitalized patients. Infect Cont Hosp Epid 2004, 25(2):164-167.

16. Hambraeus A: Transfer of Staphylococcus aureus via nurses' uniforms. J Hyg 1973, 71(4):799-814

17. Albrich WC, Harbarth S: Health-care workers: source, vector, or victim of MRSA? Lancet Infect Dis 2008, 8(5):289-301.

18. Snyder GM, Thom KA, Furuno JP, Perencevich EN, Roghmann MC, Strauss SM, Netzer G, Harris AD: Detection of methicillin-resistant Staphylococcus aureus and vancomycin-resistant enterococci on the gowns and gloves of healthcare workers. Infect Cont Hosp Epid 2008, 29(7):583-589.

19. Pittet D, Allegranzi B, Sax H, Dharan S, Pessoa-Silva CL, Donaldson L, Boyce JM, WHO Global Patient Safety Challenge WAfPS: Evidence-based model for hand 
transmission during patient care and the role of improved practices. Lancet Infect Dis 2006, 6(10):641-652.

20. Rutala WA, Weber DJ: Sterilization, high-level disinfection, and environmental cleaning. Infect Dis Clin North Am 2011, 25(1):45-76.

21. Sattar SA: Promises and pitfalls of recent advances in chemical means of preventing the spread of nosocomial infections by environmental surfaces. Am J Infect Control 2010, 38(5 Suppl 1):S34-40.

22. Verbrugh HA: Colonization with Staphylococcus aureus and the role of colonization in causing infection. In Staphylocci in human disease. 2nd edition. Edited by Crossley KB, Jefferson KK, Archer GL, Fowler VG Jr. UK: Wiley-Blackwell; 2009:255-271.

23. Davies RR, Noble WC: Dispersal of bacteria on desquamated skin. Lancet 1962, 2(7269):1295-1297.

24. Davies RR, Noble WC: Dispersal of staphylococci on desquamated skin. Lancet 1963, 1(7290):1111.

25. Clark RP, de Calcina-Goff ML: Some aspects of the airborne transmission of infection. J R Soc Interface 2009, 6(Suppl 6):S767-782.

26. Huang SS, Datta R, Platt R: Risk of acquiring antibiotic-resistant bacteria from prior room occupants. Arch Intern Med 2006, 166(18):1945-1951.

27. Meyers LA, Newman MEJ, Martin M, Schrag S: Applying network theory to epidemics: control measures for Mycoplasma pneumoniae outbreaks. Emerg Infect Dis 2003, 9(2):204-210.

28. Wang J, Wang L, Magal P, Wang Y, Zhuo J, Lu X, Ruan S: Modelling the transmission dynamics of meticillin-resistant Staphylococcus aureus in Beijing Tongren hospital. J Hosp Infect 2011, 79(4):302-308.

29. Wolkewitz M, Dettenkofer M, Bertz H, Schumacher M, Huebner J: Environmental contamination as an important route for the transmission of the hospital pathogen VRE: modeling and prediction of classical interventions. Infect Dis Res Treatment 2008, 1:3-11.

30. McBryde ES, McElwain DL: A mathematical model investigating the impact of an environmental reservoir on the prevalence and control of vancomycin-resistant enterococci. J Infect Dis 2006, 193(10):1473-1474.

31. Wang $X$, Xiao $Y$, Wang J, Lu X: A mathematical model of effects of environmental contamination and presence of volunteers on hospital infections in China. J Theor Biol 2012, 293:161-173.

32. Wilson AP, Smyth D, Moore G, Singleton J, Jackson R, Gant V, Jeanes A, Shaw $S$, James $E$, Cooper B, et al: The impact of enhanced cleaning within the intensive care unit on contamination of the near-patient environment with hospital pathogens: a randomized crossover study in critical care units in two hospitals. Crit Care Med 2011, 39(4):651-658

33. McArdle Fl, Lee RJ, Gibb AP, Walsh TS: How much time is needed for hand hygiene in intensive care? A prospective trained observer study of rates of contact between healthcare workers and intensive care patients. $J$ Hosp Infect 2006, 62(3):304-310.

34. Cookson B, Peters B, Webster M, Phillips I, Rahman M, Noble W: Staff carriage of epidemic methicillin-resistant Staphylococcus aureus. J Clin Microbiol 1989, 27(7):1471-1476.

35. Tanner El, Bullin J, Bullin CH, Gamble DR: An outbreak of post-operative sepsis due to a staphyloccoccal disperser. J Hyg 1980, 85(2):219-225.

36. Pittet D: Compliance with hand disinfection and its impact on hospitalacquired infections. J Hosp Infect 2001, 48(Suppl A):S40-46.

37. Hardy KJ, Gossain S, Henderson N, Drugan C, Oppenheim BA, Gao F, Hawkey PM: Rapid recontamination with MRSA of the environment of an intensive care unit after decontamination with hydrogen peroxide vapour. J Hosp Infect 2007, 66(4):360-368.

38. French GL, Otter JA, Shannon KP, Adams NM, Watling D, Parks MJ: Tackling contamination of the hospital environment by methicillin-resistant Staphylococcus aureus (MRSA): a comparison between conventional terminal cleaning and hydrogen peroxide vapour decontamination. J Hosp Infect 2004, 57(1):31-37.

39. Carling $P C$, Bartley JM: Evaluating hygienic cleaning in health care settings: what you do not know can harm your patients. Am J Infect Control 2010, 38(5 Suppl 1):S41-50.

40. Datta R, Platt R, Yokoe DS, Huang SS: Environmental cleaning intervention and risk of acquiring multidrug-resistant organisms from prior room occupants. Arch Intern Med 2011, 171(6):491-494.

41. Dancer SJ, White LF, Lamb J, Girvan EK, Robertson C: Measuring the effect of enhanced cleaning in a UK hospital: a prospective cross-over study. BMC Med 2009, 7:28.
42. Smith DL, Gillanders S, Holah JT, Gush C: Assessing the efficacy of different microfibre cloths at removing surface micro-organisms associated with healthcare-associated infections. J Hosp Infect 2011, 78(3):182-186.

43. Wren MW, Rollins MS, Jeanes A, Hall TJ, Coën PG, Gant VA: Removing bacteria from hospital surfaces: a laboratory comparison of ultramicrofibre and standard cloths. J Hosp Infect 2008, 70(3):265-271.

44. Moore G, Griffith C: A laboratory evaluation of the decontamination properties of microfibre cloths. J Hosp Infect 2006, 64(4):379-385.

45. Berendt AE, Turnbull L, Spady D, Rennie R, Forgie SE: Three swipes and you're out: how many swipes are needed to decontaminate plastic with disposable wipes? Am J Infect Control 2011, 39(5):442-443.

46. Cheng KL, Boost MV, Chung JW: Study on the effectiveness of disinfection with wipes against methicillin-resistant Staphylococcus aureus and implications for hospital hygiene. Am J Infect Control 2011, 39(7):577-580.

47. Williams GJ, Denyer SP, Hosein IK, Hill DW, Maillard JY: Limitations of the efficacy of surface disinfection in the healthcare setting. Infect Cont Hosp Epid 2009, 30(6):570-573.

doi:10.1186/1471-2334-13-595

Cite this article as: Plipat et al:: The dynamics of methicillin-resistant Staphylococcus aureus exposure in a hospital model and the potential for environmental intervention. BMC Infectious Diseases 2013 13:595.

\section{Submit your next manuscript to BioMed Central and take full advantage of:}

- Convenient online submission

- Thorough peer review

- No space constraints or color figure charges

- Immediate publication on acceptance

- Inclusion in PubMed, CAS, Scopus and Google Scholar

- Research which is freely available for redistribution 